\title{
Update on multiparticle effects in Bose-Einstein correlations
}

\author{
Kacper Zalewski* \\ M. Smoluchowski Institute of Physics, Jagellonian University \\ and \\ Institute of Nuclear Physics, Kraków, Poland
}

October 16, 2018

\begin{abstract}
Multiparticle effects in Bose-Einstein correlations are reviewed. It is shown that for a broad class of models they can be ignored in the low density limit, but often are significant (typically at a $10 \%$ level) for realistic final states.
\end{abstract}

PACS 25.75.Gz, 13.65.+i

Bose-Einstein correlations.

\section{Statement of the problem}

Most models of the Bose-Einstein correlations start with an input single particle density matrix $\rho_{I}\left(\mathbf{p} ; \mathbf{p}^{\prime}\right)$ or some equivalent information. The subscript $I$ reminds that this is an input and not necessarily the actual single particle density matrix of the system being considered. This input depends on the quantities of interest e.g. on the radii of the interaction region. Let us concentrate on a system of $n$ identical bosons. If these particles were distinguishable, one could build a reasonable $n$-particle density matrix as a product of the single particle density matrices $\rho_{I}$ :

${ }^{*}$ Supported in part by the KBN grant 2P03B 09322 


$$
\rho_{n}^{U}\left(\mathbf{p}_{1}, \ldots, \mathbf{p}_{n}^{\prime}\right)=\prod_{j=1}^{n} \rho_{I}\left(\mathbf{p}_{j} ; \mathbf{p}_{j}^{\prime}\right) .
$$

For identical bosons, however, this product must at least be symmetrized [1], [2] and one finds

$$
\tilde{\rho}_{n}\left(\mathbf{p}_{1}, \ldots, \mathbf{p}_{n}^{\prime}\right)=\sum_{P} \prod_{j=1}^{n} \rho_{I}\left(\mathbf{p}_{i} ; \mathbf{p}_{P i}^{\prime}\right),
$$

where the summation is over all the permutations of the momenta $\mathbf{p}_{1}^{\prime}, \ldots, \mathbf{p}_{n}^{\prime}$. The tilde reminds that in general $\operatorname{Tr} \tilde{\rho} \neq 1$ and thus, strictly speaking, $\tilde{\rho}_{n}$ is not a density matrix. This matrix is generally used to calculate the $k$-particle momentum distributions $\Omega_{k}\left(\mathbf{p}_{1}, \ldots, \mathbf{p}_{k}\right)$ putting $n=k$. Thus

$$
\begin{aligned}
\Omega_{1}(\mathbf{p}) & =\tilde{C}_{1} \rho_{I}(\mathbf{p} ; \mathbf{p}) \\
\Omega_{2}\left(\mathbf{p}_{1}, \mathbf{p}_{2}\right) & =\tilde{C}_{2}\left[\rho_{I}\left(\mathbf{p}_{1} ; \mathbf{p}_{1}\right) \rho_{I}\left(\mathbf{p}_{2} ; \mathbf{p}_{2}\right)\right. \\
\left.+\rho_{I}\left(\mathbf{p}_{1} ; \mathbf{p}_{2}\right) \rho\left(\mathbf{p}_{2} ; \mathbf{p}_{1}\right)\right] &
\end{aligned}
$$

etc., where $\tilde{C}_{j}$ are normalization constants. This is an approximation. $\Omega_{k}$ should have contributions from all $n \geq k$. A way of taking that into account has been proposed by Pratt [3]. Suppose that without symmetrization the probability of producing exactly $n$ identical bosons is $P_{0}(n)-$ this is another input. Then it is natural to assume that with symmetrization this probability goes over into

$$
P(n)=\tilde{C}_{0} P_{0}(n) \operatorname{Tr} \tilde{\rho}_{n},
$$

where by definition $\operatorname{Tr} \tilde{\rho}_{0}=1$. Formulae (3) etc. get replaced by

$$
\begin{array}{r}
\Omega_{1}(\mathbf{p})=\rho_{I}(\mathbf{p} ; \mathbf{p})+ \\
\sum_{n=2}^{\infty} P_{0}(n) \int d p_{2}, \ldots, d p_{n} \tilde{\rho}_{n}\left(\mathbf{p}_{1}, \ldots, \mathbf{p}_{n} ; \mathbf{p}_{1}, \ldots, \mathbf{p}_{n}\right),
\end{array}
$$

etc., where the normalization constants have been omitted. Depending on conventions, $d p$ may mean either $d^{3} p$ or $\frac{d^{3} p}{E_{p}}$. The difference between formulae (3) and analogous and the corresponding formulae (6) and analogous is due to the terms with integrations, or in other words to the multiparticle effects. The problem is (cf. e.g. 4], 5]): how important in practice are these multiparticle effects? 


\section{Low density limit}

Since every permutation can be decomposed into cycles, all the integrals occurring in Pratt's model can be expressed in terms of two types of basic integrals. These are the chain integrals: $G_{1}\left(\mathbf{p} ; \mathbf{p}^{\prime}\right)=\rho_{I}\left(\mathbf{p} ; \mathbf{p}^{\prime}\right)$ and for $m>1$

$$
\begin{array}{r}
G_{m}\left(\mathbf{p}, \mathbf{p}^{\prime}\right)= \\
\int \rho_{I}\left(\mathbf{p} ; \mathbf{p}_{1}\right) d p_{1} \rho_{I}\left(\mathbf{p}_{1} ; \mathbf{p}_{2}\right) \ldots d p_{m-1} \rho_{I}\left(\mathbf{p}_{m-1} ; \mathbf{p}^{\prime}\right)
\end{array}
$$

and the cycle integrals

$$
C_{m}=\int d p G_{m}(\mathbf{p} ; \mathbf{p})
$$

According to this definition $C_{1}=1$. The evaluation of the basic integrals can be greatly simplified [6], [7] when the eigenfunctions and eigenvalues of the density matrix $\rho_{I}$,

$$
\int \rho_{I}\left(\mathbf{p} ; \mathbf{p}^{\prime}\right) d p^{\prime} \psi_{k}\left(\mathbf{p}^{\prime}\right)=\lambda_{k} \psi_{k}(\mathbf{p})
$$

are known. Then

$$
\begin{aligned}
G_{m}\left(\mathbf{p} ; \mathbf{p}^{\prime}\right) & =\rho_{I}^{m}\left(\mathbf{p} ; \mathbf{p}^{\prime}\right)= \\
\sum_{k} \psi_{k}(\mathbf{p}) \lambda_{k}^{m} \psi^{*}\left(\mathbf{p}^{\prime}\right), & \\
C_{m} & =\operatorname{Tr} \rho_{I}^{m}=\sum_{k} \lambda_{k}^{m} .
\end{aligned}
$$

All the eigenvalues of a density matrix are real, nonnegative and not exceeding one. Let us label them so that

$$
0 \leq \ldots \leq \lambda_{1} \leq \lambda_{0} \leq 1
$$

The most populated state has the occupation probability (per particle) $\lambda_{0}$. Therefore, the low density limit corresponds to $\lambda_{0} \rightarrow 0$. In this limit all the basic integrals except $C_{1}$ and $G_{1}$ tend to zero and, consequently, all the integrations drop out. In the low density limit formula (3) and its more-particle analogues become exact - the multiparticle effects can be ignored. The limit of $\lambda_{0}$ is taken at constant $P_{0}(n)$. This corresponds to a constant average number of particles before symmetrization and, therefore, using the classical terminology, the density tends to zero, because the amount of available phase space grows to infinity. 
Let us consider an example. Pratt [3] chose

$$
\rho_{I}\left(\mathbf{p} ; \mathbf{p}^{\prime}\right)=\frac{1}{\sqrt{2 \pi \Delta^{2}}} e^{-\frac{\left(\mathbf{p}+\mathbf{p}^{\prime}\right)^{2}}{8 \Delta^{2}}-\frac{1}{2} R^{2}\left(\mathbf{p}-\mathbf{p}^{\prime}\right)^{2}} .
$$

For this choice [6], 7], [8]

$$
\lambda_{0}=\left(\frac{2}{1+2 \Delta R}\right)^{3} .
$$

The parameter $\Delta^{2}\left(R^{2}\right)$ is equal to the average of the square of a component of the momentum vector (position vector). Thus, in the limit $\lambda_{0} \rightarrow 0$, which corresponds to $\Delta R \rightarrow \infty$, the phase space available tends indeed to infinity.

\section{Finite density}

When $\lambda_{0}$ does not tend to zero the results become much more complicated [5]. The two-particle correlation function, which according to (3) is

$$
C_{2}\left(\mathbf{p}_{1}, \mathbf{p}_{2}\right)=1+\frac{\left|\rho_{I}\left(\mathbf{p}_{1} ; \mathbf{p}_{2}\right)\right|^{2}}{\rho_{I}\left(\mathbf{p}_{1} ; \mathbf{p}_{1}\right) \rho_{I}\left(\mathbf{p}_{2} ; \mathbf{p}_{2}\right)},
$$

becomes

$$
C_{2}\left(\mathbf{p}_{1}, \mathbf{p}_{2}\right)=C_{2}^{r e s}\left(\mathbf{p}_{1}, \mathbf{p}_{2}\right)\left[1+R_{2}\left(\mathbf{p}_{1}, \mathbf{p}_{2}\right)\right]
$$

where

$$
\begin{gathered}
C_{2}^{r e s}\left(\mathbf{p}_{1}, \mathbf{p}_{2}\right)=\frac{\sum_{i, j} w(i+j) G_{i}\left(\mathbf{p}_{1}, \mathbf{p}_{1}\right) G_{j}\left(\mathbf{p}_{2}, \mathbf{p}_{2}\right)}{\sum_{i, j} w(i) w(j) G_{i}\left(\mathbf{p}_{1}, \mathbf{p}_{1}\right) G_{j}\left(\mathbf{p}_{2}, \mathbf{p}_{2}\right)}, \\
R_{2}\left(\mathbf{p}_{1}, \mathbf{p}_{2}\right)=\frac{\sum_{i, j} w(i+j) G_{i}\left(\mathbf{p}_{1}, \mathbf{p}_{2}\right) G_{j}\left(\mathbf{p}_{2}, \mathbf{p}_{1}\right)}{\sum_{i, j} w(i+j) G_{i}\left(\mathbf{p}_{1}, \mathbf{p}_{1}\right) G_{j}\left(\mathbf{p}_{2}, \mathbf{p}_{2}\right)}, \\
w(m)=\sum_{n=m}^{\infty} P(n) \frac{n !}{(n-m) !} \frac{\operatorname{Tr} \tilde{\rho}_{n-m}}{\operatorname{Tr} \tilde{\rho}_{n}} .
\end{gathered}
$$

Function $R_{2}$ is qualitatively similar to the second term on the right-hand side of formula (15). It is equal one for $\mathbf{p}_{1}=\mathbf{p}_{2}$ and tends to zero for $\left(\mathbf{p}_{1}-\mathbf{p}_{2}\right)^{2} \rightarrow \infty$. It can be used to extract information about the geometry of the interaction region. Function $C_{2}^{\text {res }}$ has been called residual correlation [5], [9. We will see further that it is identically equal one when the input multiplicity distribution $P_{0}(n)$ is Poissonian, but in general it complicates significantly the analysis. In particular, it can make the value of $C_{2}\left(\mathbf{p}_{1}, \mathbf{p}_{2}\right)$ at large $\left(\mathbf{p}_{1}-\mathbf{p}_{2}\right)^{2}$ bigger than at $\mathbf{p}_{1}=\mathbf{p}_{2}[5]$. 
Let us consider as an example the case when the input multiplicity distribution is Poissonian with average multiplicity $\nu$ :

$$
P_{0}(n)=\frac{\nu^{n}}{n !} e^{-\nu}
$$

Then $w(m)=\nu^{m}$, which implies $w(i+j)=w(i) w(j)$, and consequently $C_{2}^{\text {res }}\left(\mathbf{p}_{1}, \mathbf{p}_{2}\right) \equiv 1$. Moreover, introducing the notation [7]

$$
L\left(\mathbf{p} ; \mathbf{p}^{\prime}\right)=\sum_{j=0}^{\infty} \nu^{j} G_{j}\left(\mathbf{p} ; \mathbf{p}^{\prime}\right),
$$

one finds [7]

$$
C_{2}\left(\mathbf{p}_{1}, \mathbf{p}_{2}\right)=1+\frac{\left.L\left(\mathbf{p}_{1} ; \mathbf{p}_{2}\right)\right|^{2}}{L\left(\mathbf{p}_{1} ; \mathbf{p}_{1}\right) L\left(\mathbf{p}_{2} ; \mathbf{p}_{2}\right)}
$$

which is formula (15) with the replacement $\rho_{I} \rightarrow L$. Similar relations hold for more-particle distributions and correlations. This analogy, however, can be misleading. Interpreting $L\left(\mathbf{p}_{1} ; \mathbf{p}_{2}\right)$ as if it were $\rho_{I}\left(\mathbf{p}_{1} ; \mathbf{p}_{2}\right)$ one overestimates the average particle multiplicity $\langle n\rangle$ and underestimates the averages $\left\langle\mathbf{p}^{2}\right\rangle$ and $\left\langle\mathbf{x}^{2}\right\rangle$. Thus, the phase space particle density is overestimated.

\section{How important is all that in practice?}

The multiparticle effects are negligible when the average population of each state, or using classical terminology the particle density in phase space, is sufficiently low. They are very important when this population, or density, is very high. The obvious question is: what about the actual data? We will present two kinds of estimates suggesting that the multiparticle corrections are likely to be of the order of $10 \%$.

\subsection{Monte Carlo approach}

Most Monte Carlo codes first generate events without Bose-Einstein correlations. These correlations are introduced later by modifying the weights of the events or by shifting the momenta of the generated particles. The former approach has a problem: the uncorrected Monte Carlo probabilities give multiplicity distributions in agreement with experiment; the weights modify the multiplicity distribution and thus tend to spoil its agreement with experiment. A way out is (cf. e.g. [10], [11]) to introduce two new parameters: $c$ and $V$ satisfying approximately the relations 


$$
P_{0}(n)=c V^{-n} P_{w}(n) .
$$

Here $P_{0}(n)$ is the uncorrected multiplicity distribution which, by construction, agrees with experiment. $P_{w}(n)$ is the distribution modified by the weight factors. A priori there is no reason why two parameters should be enough to get reasonable agreement for the full multiplicity distributions, but in practice it works. The deviation of the parameter $V$ from unity is a measure of the importance of symmetrization effects. Very roughly, it gives the change of the average particle multiplicity as result of the symmetrization. It was found that $V=1.1$ for $p p$ scattering at centre of mass energy $\sqrt{s}=30$ $\mathrm{GeV}\left[11\right.$ and $V=1.05$ for $e^{+} e^{-}$annihilations at $\sqrt{s}=172 \mathrm{GeV}$ [10]. Thus the effects are small, but not negligible when a precision of some $10 \%$ is

aimed at. These results are model dependent. It is possible to introduce weights which oscillate around one so that $V \approx 1$ [12, [13]. Symmetrization does not affect the multiplicity distribution at all in the method of momentum shifts [14], [15]. The shifts affect neither the weight of an event nor its multiplicity. In this model, however, the authors also find [15] that multiparticle effects are important, though not for the multiplicity distribution which is protected by construction. One important effect of symmetrization, common to many models, is the enhancement of the single particle momentum spectra at low momenta.

\subsection{Thermodynamics}

Many models, not only the models called thermodynamic, introduce the temperature $T$ and the chemical potential $\mu$ of the particles. In such models the momentum spectrum of the particles is given before symmetrization by the Maxwell-Boltzmann formula

$$
\Omega_{M B}(\mathbf{p})=e^{\frac{\mu}{T}} e^{-\frac{E_{p}}{T}}
$$

and after symmetrization by the Bose-Einstein formula

$$
\Omega_{B E}(\mathbf{p})=\frac{1}{e^{\frac{E_{p}-\mu}{T}}-1} .
$$

The proportionality constant in the Maxwell-Boltzmann formula has been chosen so that at large values of the particle energy $E_{p}$ the formula coincides with the Bose-Einstein one. The effect of symmetrization is the " 1 " in the denominator of the Bose-Einstein formula. The first remark is that this correction becomes more important with respect to the exponential when 
temperature increases. Incidentally, for this reason some people consider Einstein's condensation a high temperature effect.

Temperatures in high energy multiple particle production processes are strongly model dependent. Heavy ion collisions models where all transverse momentum is due to thermal motion have $T \approx\left\langle p_{t}\right\rangle \approx 350 \mathrm{MeV}$. Models where much of the transverse momenta is due to collective radial expansion have lower (local) temperatures of about $100 \mathrm{MeV}$. The chemical potential cannot exceed the lowest possible energy, in this case $m_{\pi}=140 \mathrm{MeV}$. Fits assuming temperatures of about $100 \mathrm{MeV}$ [16], [17] give values of $\mu$ ranging from $30 \mathrm{MeV}$ to $90 \mathrm{MeV}$. We will give two estimates of the importance of multiparticle effects based on these numbers.

The symmetrization effects are the strongest at $\mathbf{p}=\mathbf{0}$. The ratio of the Bose-Einstein to the Maxwel-Boltznann distribution at $\mathbf{p}=\mathbf{0}$ is

$$
r_{1}=\frac{1}{1-e^{-\frac{m_{\pi}-\mu}{T}}}
$$

For $T=100 \mathrm{MeV}$ and $\mu$ ranging from $30 \mathrm{MeV}$ to $90 \mathrm{MeV} r_{1}$ ranges from 1.5 to 2.5 . Thus the correction is very significant. A more representative ratio is the ratio of the integrated multiplicities corresponding to the two distributions:

$$
r_{2}=\frac{\int p^{2} \Omega_{B E}(\mathbf{p}) d p}{\int p^{2} \Omega_{M B}(\mathbf{p}) d p} .
$$

For the same choice of parameters $r_{2}$ ranges from 1.09 to 1.20 , which is consistent with the Monte Carlo results.

\section{Conclusions}

The importance of the multiparticle effects depends both on the model being used and on the quantities of interest. In Monte Carlo models, fine tuned to reproduce without symmetrization the multiplicity distributions, symmetrization is forbidden to affect the multiplicity distributions. In models related to thermodynamics the low momentum particles are affected much more than the high momentum particles. It is not unusual to get from multiparticle effects corrections of the order of $10 \%$ or more. Therefore, it seems that in precision work the multiparticle effects should not be discarded without previous analysis. 


\section{References}

[1] J. Karczmarczuk, Nucl. Phys. B78(1974)370.

[2] A. Bialas and A. Krzywicki, Phys. Lett. B354(1995)134.

[3] S. Pratt, Phys. Lett. B301(1993)159.

[4] W.A. Zajc, Phys. Rev. D33(1987)3396.

[5] U. Heinz, P.Scotto and Q.H. Zhang, Ann. of Phys. 288(2001)325.

[6] A. Bialas and K. Zalewski, Phys. Lett. B463(1998)153.

[7] A. Bialas and K. Zalewski, Eur. Phys. J. C6(1999)349.

[8] K. Zalewski, Acta Phys. Pol. B33(2002)1361.

[9] W.A. Zajc et al., Phys. Rev. C29(1984)2173.

[10] S. Jadach and K. Zalewski, Acta Phys. Pol. B28(1997)1363.

[11] K. Fiałkowski and R. Wit, Eur. J. Phys.C2(1998)691.

[12] V. Kartvelishvili and R. Kvatadze, Phys. Lett.B514(2001)7.

[13] V. Karetvelishvili, private communication.

[14] L. Lönnblad and T. Sjöstrand, Phys. Lett. B351(1995)293.

[15] L. Lönnblad and T. Sjöstrand, Eur. Phys. J. C2(1998)165.

[16] B. Tomasik and U.A. Wiedemann, Phys. Rev. C68(2003)034905.

[17] S.V. Akkelin and Yu. M. Sinyukov, nucl-th/0310036. 